
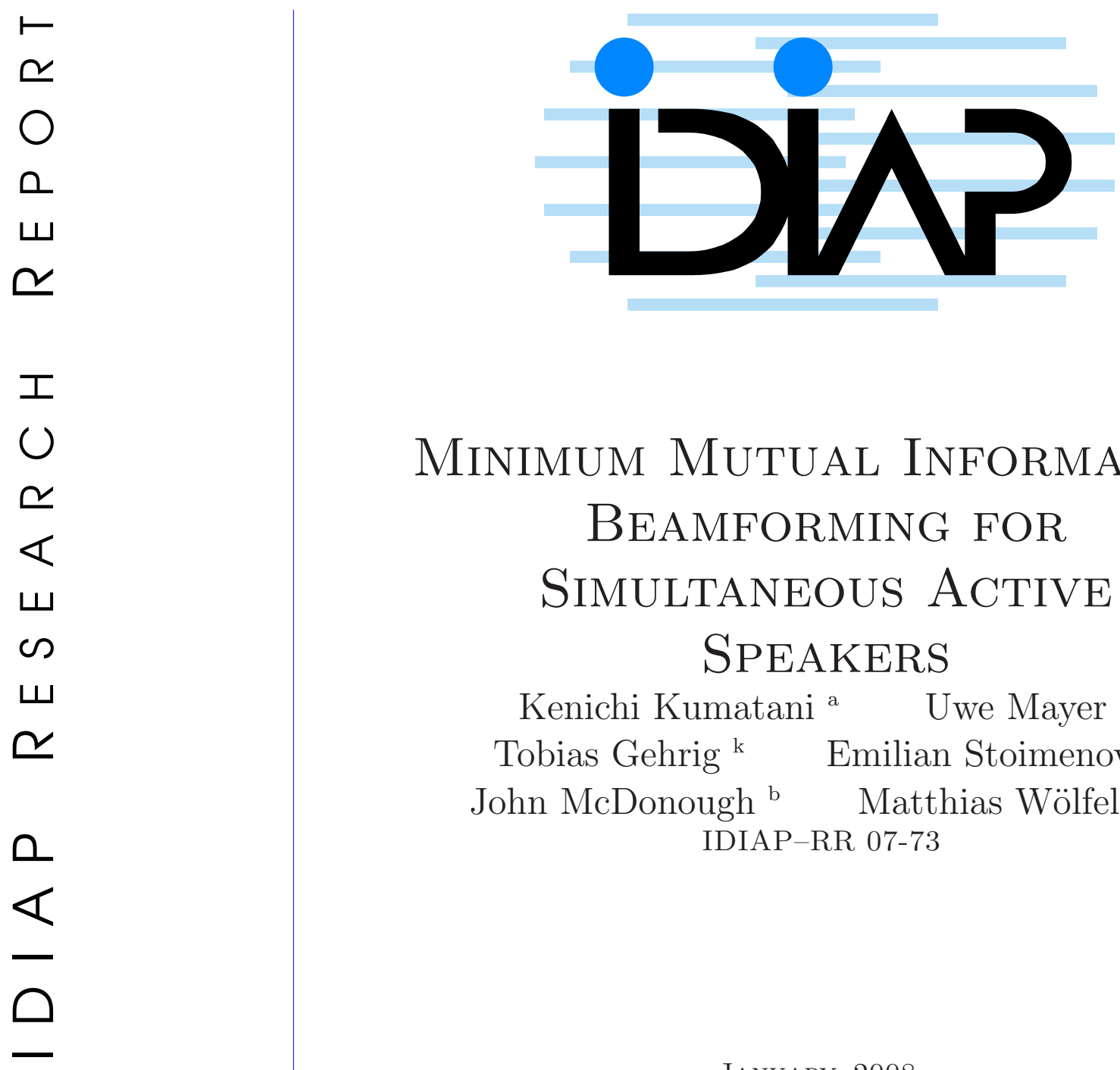

\title{
Minimum Mutual Information BEAMFORMING FOR Simultaneous Active SPEAKERS
}

\author{
Kenichi Kumatani a Uwe Mayer ${ }^{k}$ \\ Tobias Gehrig ${ }^{k} \quad$ Emilian Stoimenov ${ }^{k}$ \\ John McDonough $^{\mathrm{b}} \quad$ Matthias Wölfel ${ }^{\mathrm{k}}$ \\ IDIAP-RR 07-73
}

JANUARY 2008

a IDIAP Research Institute, Martigny, Switzerland

b Spoken Language Systems at Saarland University in Saarbrücken, Germany

k Institute for Theoretical Computer Science at University of Karlsruhe in Karlsruhe, Germany 

IDIAP Research Report 07-73

\title{
Minimum Mutual Information Beamforming for Simultaneous Active Speakers
}

\author{
Kenichi Kumatani Uwe Mayer \\ Tobias Gehrig \\ Emilian Stoimenov \\ John McDonough \\ Matthias Wölfel
}

JANUARY 2008

\begin{abstract}
In this work, we consider an acoustic beamforming application where two speakers are simultaneously active. We construct one subband-domain beamformer in generalized sidelobe canceller (GSC) configuration for each source. In contrast to normal practice, we then jointly optimize the active weight vectors of both GSCs to obtain two output signals with minimum mutual information (MMI). Assuming that the subband snapshots are Gaussian-distributed, this MMI criterion reduces to the requirement that the cross-correlation coefficient of the subband outputs of the two GSCs vanishes. We also compare separation performance under the Gaussian assumption with that obtained from several super-Gaussian probability density functions (pdfs), namely, the Laplace, $K_{0}$, and $\Gamma$ pdfs. Our proposed technique provides effective nulling of the undesired source, but without the signal cancellation problems seen in conventional beamforming. Moreover, our technique does not suffer from the source permutation and scaling ambiguities encountered in conventional blind source separation algorithms. We demonstrate the effectiveness of our proposed technique through a series of far-field automatic speech recognition experiments on data from the PASCAL Speech Separation Challenge (SSC). On the SSC development data, the simple delay-and-sum beamformer achieves a word error rate (WER) of $70.4 \%$. The MMI beamformer under a Gaussian assumption achieves a $55.2 \%$ WER, which is further reduced to $52.0 \%$ with a $K_{0}$ pdf, whereas the WER for data recorded with a close-talking microphone is $21.6 \%$.
\end{abstract}




\section{Introduction}

In acoustic beamforming, it is typically assumed that the position of the speaker is estimated by a speaker localization system. A conventional beamformer in generalized sidelobe canceller (GSC) configuration is structured such that the direct signal from the speaker is undistorted [1, §6.7.3]. Subject to this distortionless constraint, the total output power of the beamformer is minimized through the appropriate adjustment of an active weight vector, which effectively places a null on any source of interference, but can also lead to an undesirable signal cancellation. To avoid the latter, the adaptation of the active weight vectors is typically halted whenever the desired source is active.

In this work, we consider a situation where two speakers are simultaneously active. We construct one subband domain beamformer GSC configuration for each source. In contrast to normal practice, we then jointly adjust the active weight vectors of both GSCs to obtain two output signals with minimum mutual information (MMI). Parra and Alvino [2] proposed a geometric source separation (GSS) algorithm with similarities to the algorithm proposed here. Their algorithm attempts to decorrelate the outputs of two beamformers. We discuss Parra and Alvino's GSS algorithm in Section 3.3, and propose novel algorithms which assume the probability density function (pdf) of subband snapshots are Gaussian and super-Gaussian.

In the case that the Gaussian assumption is applied, our system has the same criterion proposed in [3] except that the cross-power spectral density is recursively updated in [3] though we calculate it over the utterance. The critical difference between our and their algorithms is that we update only active weight vectors while keeping the same quiescent vectors. Therefore, the response of the mainlobe is always kept unity gain in the desired look direction.

We demonstrate the effectiveness of our proposed technique through a series of far-field automatic speech recognition experiments on data from the PASCAL Speech Separation Challenge (SSC). As this data was recorded from actual speakers in a real, reverberant room, it provides the possibility of conducting source separation experiments under realistic conditions, which is noteably different from the vast majority of the experiments reported in the beamforming and blind source separation literature.

The balance of this work is organized as follows. In Section 2, we review the definition of mutual information, and demonstrate that, under a Gaussian assumption, the mutual information of two complex random variables is a simple function of their cross-correlation coefficient. We discuss our MMI beamforming criterion in Section 3, and compare it to the approach of Parra and Alvino [2]. Section 4 presents the framework needed to apply minimum mutual information beamforming when the Gaussian assumption is relaxed. In particular, we develop multivariate pdfs for the Laplace, $K_{0}$ and $\Gamma$ density functions, and then develop parameter estimation formulae based on these for optimizing the active weight vector of a GSC. In Section 5, we present the results of far-field automatic speech recognition experiments conducted on data from the PASCAL Speech Separation Challenge; see Lincoln et al. [4] for a description of the data collection apparatus. Finally, in Section 6, we present our conclusions and plans for future work.

\section{Mutual Information}

Here we derive the mutual information of two zero-mean Gaussian random variables (r.v.s).

Consider two r.v.s $Y_{1}$ and $Y_{2}$. By definition, the mutual information [5] of $Y_{1}$ and $Y_{2}$ can be expressed as

$$
I\left(Y_{1}, Y_{2}\right)=\mathcal{E}\left\{\log \frac{p\left(Y_{1}, Y_{2}\right)}{p\left(Y_{1}\right) p\left(Y_{2}\right)}\right\}
$$

where $\mathcal{E}\{\}$ indicates the ensemble expectation.

The univariate Gaussian pdf for complex r.v.s $Y_{i}$ can be expressed as

$$
p\left(Y_{i}\right)=\frac{1}{\pi \sigma_{i}^{2}} \exp \left(-\left|Y_{i}\right|^{2} / \sigma_{i}^{2}\right)
$$


where $\sigma_{i}^{2}=\mathcal{E}\left\{Y_{i} Y_{i}^{*}\right\}$ is the variance of $Y_{i}$. Let us define the zero-mean complex random vector $\mathbf{Y}=\left[\begin{array}{ll}Y_{1} & Y_{2}\end{array}\right]^{T}$ and the covariance matrix.

$$
\Sigma_{Y}=\mathcal{E}\left\{\mathbf{Y} \mathbf{Y}^{H}\right\}=\left[\begin{array}{cc}
\sigma_{1}^{2} & \sigma_{1} \sigma_{2} \rho_{12} \\
\sigma_{1} \sigma_{2} \rho_{12} & \sigma_{2}^{2}
\end{array}\right]
$$

where

$$
\rho_{12}=\frac{\epsilon_{12}}{\sigma_{1} \sigma_{2}} \text { and } \epsilon_{12}=\mathcal{E}\left\{Y_{1} Y_{2}^{*}\right\}
$$

The bivariate Gaussian pdf for complex r.v.s is given by

$$
p\left(Y_{1}, Y_{2}\right)=\frac{1}{\pi^{2}\left|\Sigma_{Y}\right|} \exp \left(-\mathbf{Y}^{T} \Sigma_{Y}^{-1} \mathbf{Y}\right)
$$

It follows that the mutual information (1) for jointly Gaussian complex r.v.s can be expressed as [6]

$$
I\left(Y_{1}, Y_{2}\right)=-\frac{1}{2} \log \left(1-\left|\rho_{12}\right|^{2}\right)
$$

From (5), it is clear that minimizing the mutual information between two zero-mean Gaussian r.v.s is equivalent to minimizing the magnitude of their cross correlation coefficient $\rho_{12}$, and that $I\left(Y_{1}, Y_{2}\right)=0$ if and only if $\left|\rho_{12}\right|=0$.

\section{Beamforming}

Consider a subband beamformer in GSC configuration as shown in Figure 1. Assuming there are two such beamformers aimed at different sources, the output of the $i$-th beamformer for a given subband can be expressed as,

$$
Y_{i}=\left(\mathbf{w}_{q, i}-\mathbf{B}_{i} \mathbf{w}_{a, i}\right)^{H} \mathbf{X}
$$

where $\mathbf{w}_{q, i}$ is the quiescent weight vector for the $i$-th source, $\mathbf{B}_{i}$ is the blocking matrix, $\mathbf{w}_{a, i}$ is the active weight vector, and $\mathbf{X}$ is the input subband snapshot vector. In keeping with the GSC formalism, $\mathbf{w}_{q, i}$ is chosen to preserve a signal from the look direction and, at the same time, to suppress an interference [1, §6.3]. $\quad \mathbf{B}_{i}$ is chosen such that $\mathbf{B}_{i}^{H} \mathbf{w}_{q, i}=\mathbf{0}$. The active weight vector $\mathbf{w}_{a, i}$ is typically chosen to maximize the signal-to-noise ratio (SNR). Here, however, we develop an optimization procedure to find that $\mathbf{w}_{a, i}$ which minimizes the mutual information $I\left(Y_{1}, Y_{2}\right)$. Minimizing a mutual information criterion yields a weight vector $\mathbf{w}_{a, i}$ capable of canceling interference that leaks through the sidelobes without the signal cancellation problems encountered in conventional beamforming.

The subband analysis and resynthesis can be performed with a perfect reconstruction filterbank such as the popular cosine modulated filterbank $[7, \S 8]$. Beamforming in the subband domain has the considerable advantage that the active sensor weights can be optimized for each subband independently, which saves a tremendous computation. In addition, the GSC constraint solves the problems with source permutation and scaling ambiguity typically encountered in conventional blind source separation algorithms [8].

\subsection{Parameter Optimization}

In the absence of a closed-form solution for those $\mathbf{w}_{a, i}$, we must use a numerical optimization algorithm. Such an optimization algorithm typically requires gradient information. We used a conjugate gradient algorithm to obtain active filter vectors $\mathbf{w}_{a}$ which provides minimum mutual information $[9, \S 1.6]$. The detail is reported in [6]. 


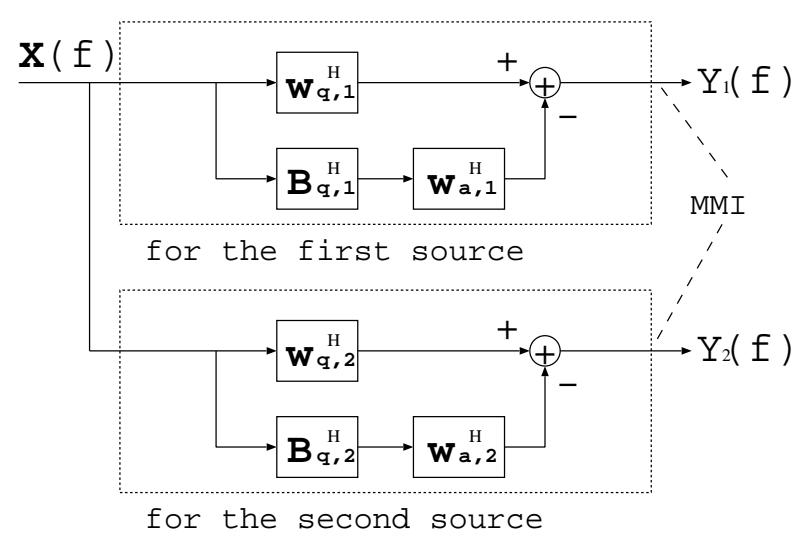

Figure 1: A beamformer in GSC configuration.

\subsection{Regularization}

In conventional beamforming, a regularization term is often applied that penalizes large active weights, and thereby improves robustness by inhibiting the formation of excessively large sidelobes $[1, \S 6.10]$. Such a regularization term can be applied in the present instance by defining the modified optimization criterion

$$
\mathcal{I}\left(Y_{1}, Y_{2} ; \alpha\right)=I\left(Y_{1}, Y_{2}\right)+\alpha\left\|\mathbf{w}_{a, 1}\right\|^{2}+\alpha\left\|\mathbf{w}_{a, 2}\right\|^{2}
$$

for some real $\alpha>0 . \alpha=0.01$ is set in this experiment.

\subsection{Geometric Source Separation}

Parra and Alvino [2] proposed a geometric source separation (GSS) algorithm which has many similarities to the proposed algorithm. Instead of minimizing the mutual information between two signals, Parra and Alvino sought to diagonalize the cross-power spectra under geometric constraints which are equivalent to the distortionless constraint inherent in the GSC. In the case of a Gaussian pdf, the principal difference between GSS and the algorithm proposed here, is that GSS seeks to minimize $\left|\epsilon_{12}\right|^{2}$ instead of $\left|\rho_{12}\right|^{2}$. Although the difference between minimizing $\left|\epsilon_{12}\right|^{2}$ instead of $\left|\rho_{12}\right|^{2}$ may seem very slight, it can in fact lead to radically different behavior. To achieve the desired optimum, both criteria will seek to place deep nulls on the unwanted source; this characteristic is associated with $\left|\epsilon_{12}\right|^{2}$, which also comprises the numerator of $\left|\rho_{12}\right|^{2}$. Such null steering is also observed in conventional adaptive beamformers $[1, \S 6.3]$. The difference between the two optimization criteria is due to the presence of the terms $\sigma_{i}^{2}$ in the denominator of $\left|\rho_{12}\right|^{2}$, which indicate that, in addition to nulling out the unwanted signal, improvements are possible by increasing the strength of the desired signal. In acoustic beamforming in realistic environments, there are typically strong reflections from hard surfaces such as tables and walls. A conventional beamformer would attempt to null out all such strong reflections. The GSS algorithm would attempt to null out those reflections from the unwanted signal. But in addition to nulling out reflections from the unwanted signal, the MMI beamforming algorithm would attempt to strengthen those reflections from the desired source; assuming statistically independent sources, strengthening a reflection from the desired source would have little or no effect on the numerator of $\left|\rho_{12}\right|^{2}$, but would increase the denominator, thereby leading to an overall reduction of optimization criterion. Of course, any reflected signal would be delayed with respect to the direct path signal. Such a delay would, however, manifest itself as a phase shift in the subband domain, and could thus be removed through a suitable choice of $\mathbf{w}_{a}$. Hence, the MMI beamformer offers the possibility of steering both nulls and sidelobes; the former towards the undesired signal and its reflections, the latter towards reflections of the desired signal. 


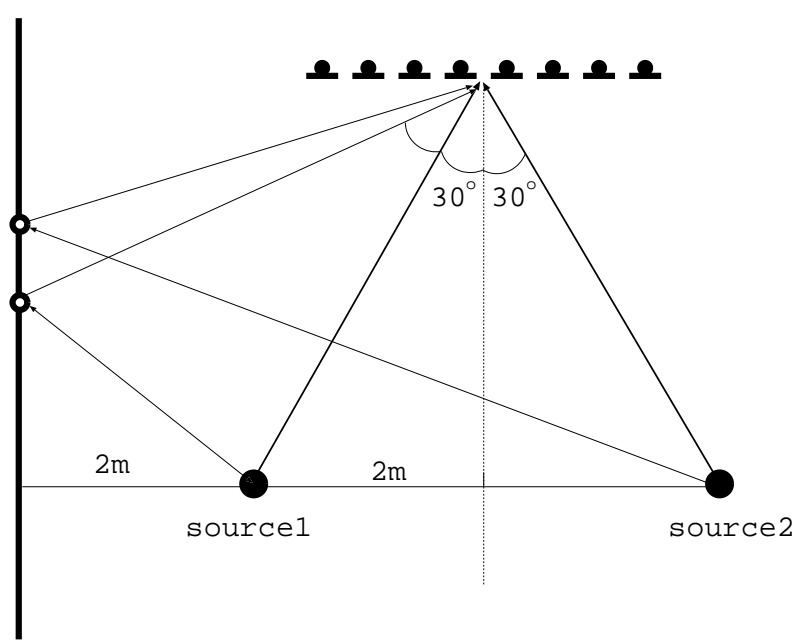

Figure 2: Configuration of the simulation environment.

\begin{tabular}{|c||c|}
\hline pdf & $\frac{1}{T} \sum_{t=0}^{T-1} \log p\left(X_{t} ; \mathrm{pdf}\right)$ \\
\hline$\Gamma$ & -0.779 \\
$K_{0}$ & -1.11 \\
Laplace & -2.48 \\
Gaussian & -9.93 \\
\hline
\end{tabular}

Table 1: Average log-likelihoods of subband speech samples for various pdfs.

In order to verify that the MMI beamforming algorithm forms sidelobes directed towards the reflections of a desired signal, we conducted experiments with a simulated acoustic environment. As shown in Figure 2, we considered a simple configuration where there are two sound sources, a reflective surface, and an eight-channel linear microphone array that captures both the direct and reflected waves from each source. Actual speech data were used as sound sources in this simulation, which was based on the image method [10].

Figure 3 shows beam patterns at fs $=1500 \mathrm{~Hz}$ and fs $=3000 \mathrm{~Hz}$ obtained with the MMI beamformer and the GSS algorithm. In order to make the techniques directly comparable, the implementation of the GSS algorithm used for the simulation was based on two GSCs, each aimed at one target. Both MMI beamformer and GSS algorithm formed the beam patterns so that the signal from Source 2 in Figure 2 was enhanced while the other from Source 1 was suppressed. It is clear that both algorithms have unity gain in the look direction, and place deep nulls on the direct path of the unwanted source. The suppression of Reflection 1, the undesired interference, by the MMI beamformer is equivalent to or better than that provided by the GSS algorithm for both frequencies. Moreover, the enhancement of Reflection 2, the desired signal, by the MMI beamformer is stronger than that of the GSS algorithm.

\section{Super-Gaussian Probability Density Functions}

In the field of independent component analysis (ICA), it is common practice to use mutual information as a measure of the independence of two or more signals as in the prior sections. The entire field of ICA, however, is founded on the assumption that all signals of real interest are not Gaussian-distributed. A concise and very readable argument for the validity of this assumption is given by Hyvärinen and Oja [5].

Table 1 shows the average log-likelihood of subband samples of speech recorded with a close- 
IDIAP-RR 07-73
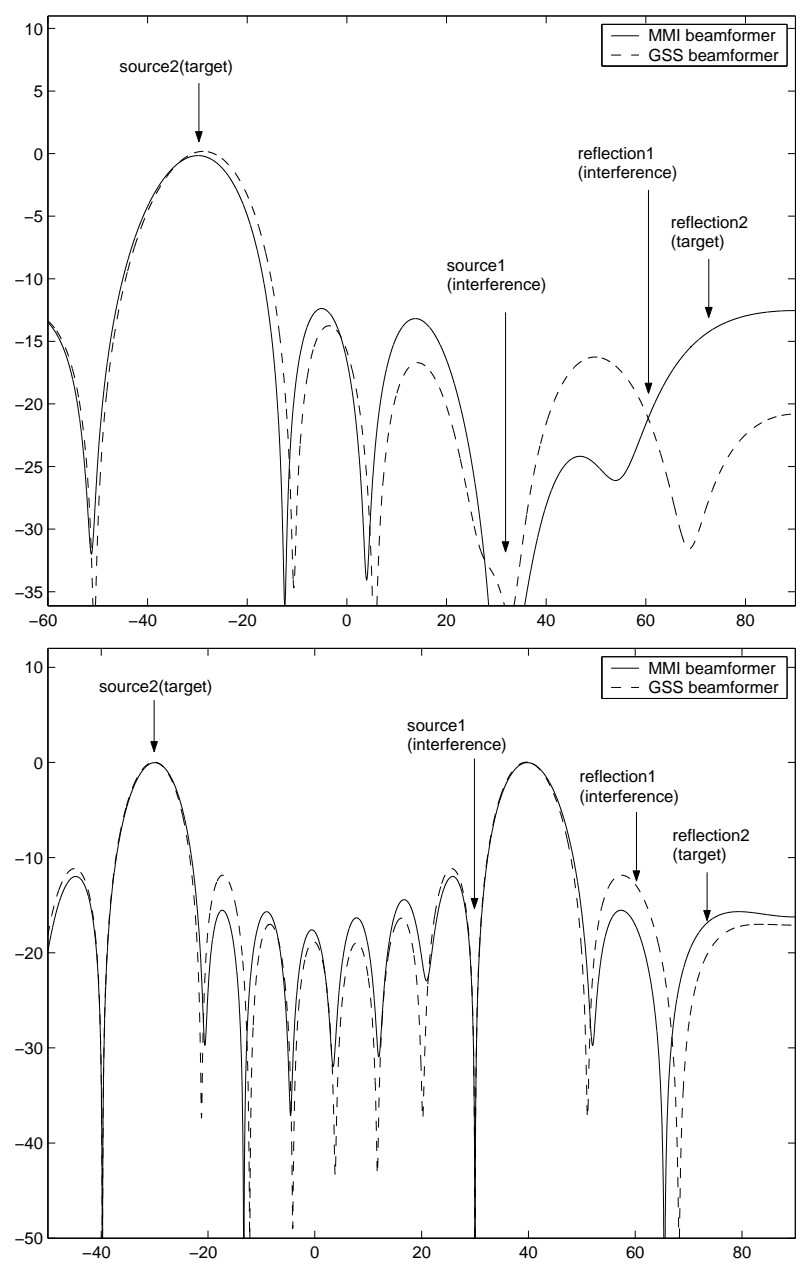

Figure 3: Beam patterns produced by the MMI beamformer and GSS algorithm using a spherical wave assumption for (a) fs $=1500 \mathrm{~Hz}$ and (b) fs $=3000 \mathrm{~Hz}$.

talking microphone (CTM) as calculated with the Gaussian and three super-Gaussian pdfs, namely, the Laplace, $K_{0}$ and $\Gamma$ pdfs. It is clear from these log-likelihood values that the complex subband samples of speech are in fact better modelled by the super-Gaussian pdfs considered here than the Gaussian. Hence, the abstract arguments on which the field of ICA are founded correspond well to the actual characteristics of speech.

A plot of the log-likelihood of the Gaussian and three super-Gaussian real univariate pdfs considered here is provided in Figure 4. From the figure, it is clear that the Laplace, $K_{0}$ and $\Gamma$ densities exhibit the "spikey" and "heavy-tailed" characteristics that are typical of super-Gaussian pdfs. This implies that they have a sharp concentration of probability mass at the mean, relatively little probability mass as compared with the Gaussian at intermediate values of the argument, and a relatively large amount of probability mass in the tail; i.e., far from the mean.

The kurtosis of a r.v. $Y$, defined as

$$
\operatorname{kurt}(Y)=\mathcal{E}\left\{Y^{4}\right\}-3\left(\mathcal{E}\left\{Y^{2}\right\}\right)^{2}
$$

is a measure of how non-Gaussian it is [5]. The Gaussian pdf has zero kurtosis; pdfs with positive kurtosis are super-Gaussian; those with negative kurtosis are sub-Gaussian. Of the three superGaussian pdfs considered here, the $\Gamma$ pdf has the highest kurtosis, followed by the $K_{0}$, then by the 


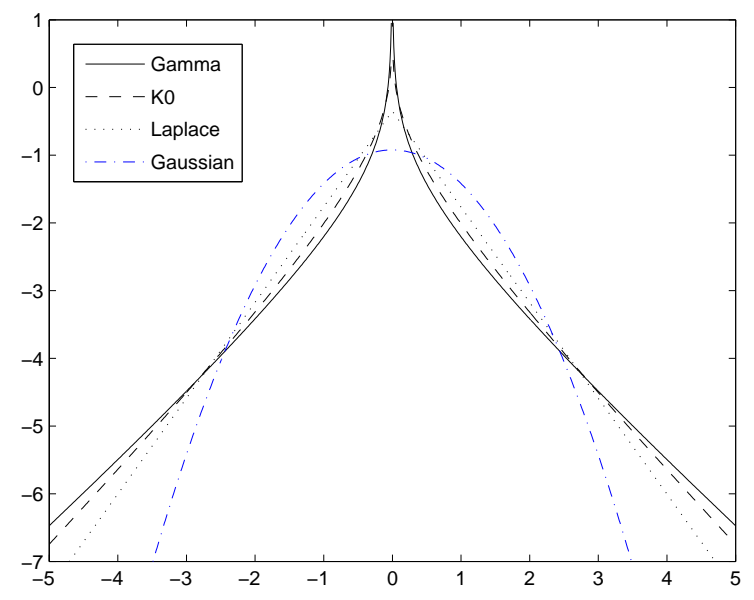

Figure 4: Plot of the log-likelihood of the super-Gaussian and Gaussian pdfs.

Laplace pdf. This fact manifests itself in Figure 4, where it is clear that as the kurtosis increases, the pdf becomes more and more spikey and heavy-tailed. It is also clear from Table 1 that the average log-likelihood of the subband samples of speech improves significantly as the kurtosis of the pdf used to measure the log-likelihood increases. This is a further proof of the validity of the assumptions on which ICA is based for speech processing.

As explained in Brehm and Stammler [11], Laplace, $K_{0}$ and $\Gamma$ density pdfs belong to the class of spherically invariant random processes (SIRPs), which is a very attractive feature for two reasons. Firstly, it implies that multivariate pdfs of all orders can be readily derived from the theory of Meijer G-functions [12] based solely on the knowledge of the covariance matrix of the random vectors. Secondly, such variates can be extended to the case of complex r.v.s, which is essential for our current development.

For complex Laplace r.v.s $Y_{i} \in \mathbf{C}$, the univariate pdf can be expressed as

$$
p_{\text {Lap }}\left(Y_{i}\right)=\frac{4}{\sqrt{\pi} \sigma_{Y}^{2}} K_{0}\left(\frac{2 \sqrt{2}\left|Y_{i}\right|}{\sigma_{Y}}\right)
$$

where $K_{0}(z)$ is the modified Bessel function of the second kind Mathematica $[13, \S 3.2 .10]$ and $\sigma_{Y}^{2}=$ $\mathcal{E}\left\{\left|Y_{i}\right|^{2}\right\}$. For $\mathbf{Y} \in \mathbf{C}^{2}$, the bivariate Laplace pdf is given by

$$
p_{\text {Lap }}(\mathbf{Y})=\frac{16}{\pi^{3 / 2}\left|\Sigma_{\mathbf{Y}}\right| \sqrt{s}} K_{1}(4 \sqrt{s})
$$

where

$$
\Sigma_{\mathbf{Y}}=\mathcal{E}\left\{\mathbf{Y} \mathbf{Y}^{H}\right\} \text { and } s=\mathbf{Y}^{H} \Sigma_{\mathbf{Y}}^{-1} \mathbf{Y}
$$

Similarly, we can write the univarite $K_{0}$ pdf for complex r.v.s $Y_{i} \in \mathbf{C}$ as

$$
p_{K_{0}}\left(Y_{i}\right)=\frac{1}{\sqrt{\pi} \sigma_{Y}\left|Y_{i}\right|} \exp \left(-2\left|Y_{i}\right| / \sigma_{Y}\right)
$$

The bivariate $K_{0}$ pdf for $\mathbf{Y} \in \mathbf{C}^{2}$ can be expressed as

$$
p_{K_{0}}(\mathbf{Y})=\frac{\sqrt{2}+4 \sqrt{s}}{2 \pi^{3 / 2}\left|\Sigma_{\mathbf{Y}}\right| s^{3 / 2}} \exp (-2 \sqrt{2 s})
$$


Those formulas are different from the forms of the real univariate pdfs because those are derived from the Meijer G-functions and extended for a complex valued vector. Derivations of (8-11) are provided in [6]. For the $\Gamma$ pdf, the complex univariate and bivariate pdfs cannot be expressed in closed form in terms of elementary or even special functions. However, it is possible to derive Taylor series expansions that enable the required variates to be calculated to arbitrary accuracy [6].

The mutual information can no longer be expressed in closed form as in (5) for the super-Gaussian pdfs. We can, however, replace the exact mutual information with the empirical mutual information

$$
\begin{aligned}
I\left(Y_{1}, Y_{2}\right) \approx \frac{1}{N} & \sum_{t=0}^{N-1}\left[\log p\left(\mathbf{Y}^{(t)}\right)\right. \\
& \left.-\sum_{i=1}^{2} \log p\left(Y_{i}^{(t)}\right)\right]
\end{aligned}
$$

Such an empirical approximation was used for the experiments described in the next section.

\section{Experiments}

We performed far-field automatic speech recognition experiments on development data from the $P A S$ CAL Speech Separation Challenge (SSC) [4]. The data contain recordings of five pairs of speakers and each pair of speakers reads approximately 30 sentences taken from the 5,000 word vocabulary Wall Street Journal (WSJ) task. The data were recorded with two circular, eight-channel microphone arrays. The diameter of each array was $20 \mathrm{~cm}$, and the sampling rate of the recordings was $16 \mathrm{kHz}$. The database also contains speech recorded with close talking microphones (CTM). This is a challenging task for source separation algorithms given that the room is reverberant and some recordings include significant amounts of background noise. In addition, as the recorded data is real and not artificially convoluted with measured room impulse responses, the position of the speaker's head as well as the speaking volume varies.

The directivity of the circular array at low frequencies is poor; this stems from the fact that for low frequencies, the wave is much longer than the aperture of the array. At high frequencies, the beam pattern is characterized by very large sidelobes; this is due to the fact that at high frequencies, the spacing between the elements of the array exceeds half the length of the wave, thereby causing spatial aliasing $[1, \S 2.5]$.

Prior to beamforming, we first estimated the speaker's position with the speaker localization system described in [14]. In addition to the speaker position, our source localization system is also capable of determining when each source is active. This information proved very useful to segment the utterance of each speaker, given that the utterance spoken by one speaker was often much longer than that spoken by the other. In the absence of perfect separation, which we could not achieve with the algorithms described here, running the speech recognizer over the entire waveform from the beamformer instead of only that portion where a given speaker was actually active would have resulted in significant insertion errors. These insertions would also have proven disastrous for speaker adaptation, as the adaptation data from one speaker would have been contaminated with speech of the other speaker.

The active weights for each subband were initialized to zero for estimation with the Gaussian pdf. For estimation with the super-Gaussian pdfs, the active weights were initialized to the optimal values under the Gaussian assumption.

After beamforming, the feature extraction of our ASR system was based on cepstral features estimated with a warped minimum variance distortionless response [15] (MVDR) spectral envelope of model order 30. We concatenated 15 cepstral features, each of length 20, then applied linear discriminant analysis (LDA) [16, §10] and a semi-tied covariance (STC) [17] transform to obtain final features of length 42 for speech recognition. The far-field ASR experiments reported here were conducted entirely with the Millenium automatic speech recognition system. Millenium is based 


\begin{tabular}{|c||c|c|c|c|}
\hline \multicolumn{1}{|c||}{ Beamforming } & \multicolumn{4}{c|}{ Pass (\%WER) } \\
\cline { 2 - 5 } Algorithm & 1 & 2 & 3 & 4 \\
\hline Delay \& Sum & 85.1 & 77.6 & 72.5 & 70.4 \\
GSS & 80.1 & 65.5 & 60.1 & 56.3 \\
MMI: Gaussian & 79.7 & 65.6 & 57.9 & 55.2 \\
MMI: Laplace & 81.1 & 67.9 & 59.3 & 53.8 \\
MMI: $K_{0}$ & 78.0 & 62.6 & 54.1 & 52.0 \\
MMI: $\Gamma$ & 80.3 & 63.0 & 56.2 & 53.8 \\
CTM & 37.1 & 24.8 & 23.0 & 21.6 \\
\hline
\end{tabular}

Table 2: Word error rates for every beamforming algorithm after every decoding passes.

on the Enigma weighted finite-state transducer (WFST) library, which contains implementations of all standard WFST algorithms, including weighted composition, weighted determinization, weight pushing, and minimization. The word trace decoder in Millenium is implemented along the lines suggested by Saon et al. [18], and is capable of generating word lattices, which can then be optimized with WFST operations as in [19].

The training data used for the experiments were taken from the ICSI, NIST, and CMU meeting corpora, as well as the Transenglish Database (TED) corpus, for a total of 100 hours of training material. In addition to these corpora, approximately 12 hours of speech from the WSJCAM0 corpus [20] was used for HMM training in order to cover the British accents for the speakers [4]. Acoustic models estimated with three different HMM training schemes were used for the several decoding passes: conventional maximum likelihood (ML) HMM training [21, §12], speaker-adapted training under a ML criterion (ML-SAT) [22]. Our baseline system was fully continuous with 3,500 codebooks and a total of 180,656 Gaussian components.

We performed four passes of decoding on the waveforms obtained with each of the beamforming algorithms. Parameters for speaker adaptation were estimated using the word lattices generated during the prior pass as in [23]. A description of the individual decoding passes follows:

1. Decode with the unadapted, conventional ML acoustic model and bigram language model (LM).

2. Estimate vocal tract length normalization (VTLN) [24] parameters and constrained maximum likelihood linear regression parameters (CMLLR) [25] for each speaker, then redecode with the conventional ML acoustic model and bigram LM.

3. Estimate VTLN, CMLLR, and maximum likelihood linear regression (MLLR) [26] parameters for each speaker, then redecode with the ML-SAT model and bigram LM.

4. Estimate VTLN, CMLLR, MLLR parameters, then redecode with the ML-SAT model and bigram LM.

Table 2 shows the word error rate (WER) for every beamforming algorithm and speech recorded with the CTM after every decoding pass on the SSC data. After the fourth pass, the delay-and-sum beamformer has the worst recognition performance of $70.4 \%$ WER. This is not surprising given that the mixed speech was not well separated by the delay-and-sum beamformer for the reasons mentioned above. The MMI beamformer with a Gaussian pdf (55.2\%) was somewhat better than the GSS algorithm $(56.3 \%)$, which is what should be expected given the reasoning in Section 3.3. The best performance was achieved with the $K_{0}$ pdf assumption $(52.0 \%)$.

Although $\Gamma$ pdf assumption gave the highest log-likelihood, as reported in Table 1 , the $K_{0}$ pdf achieved the best recognition performance. There are several possible explanations for this: Firstly, as mentioned in Section 6, the subband filter bank used for the experiments reported here may not be optimally suited for beamforming and adaptive filtering applications [27]. Hence, aliasing introduced by the filter bank could be masking the gain which would otherwise be obtained by using a pdf with 
higher kurtosis to calculate mutual information and optimize the active weight vectors. Secondly, data recorded in the real environments contains background noise as well as speech. If the pdf of the noise is super-Gaussian, it could conceivably be emphasized by the MMI beamformer with a super-Gaussian pdf assumption. Feature and model adaptation algorithms such as CMLLR and MLLR can, however, robustly estimate parameters to compensate for the background noise. As a result, such an effect is mitigated by the speaker adaptation. From Table 2, this is evident from the significant improvement after the second pass when the $\Gamma$ pdf is used; to wit, the results obtained with the $\Gamma$ pdf go from being somewhat worse than the Gaussian results after the first unadapted pass to significantly better after the second pass with VTLN and CMLLR adaptation, and remain significantly better after all subsequent adapted passes.

\section{Conclusions and Future Work}

In this work, we have proposed a novel beamforming algorithm for simultaneous active speakers based on minimizing mutual information. The proposed method does not exhibit the signal cancellation problems typically seen in conventional adaptive beamformers. Moreover, unlike conventional BSS techniques, the proposed algorithm does not have permutation and scaling ambiguities that cause distortions in the output speech. We evaluated the Gaussian and three super-Gaussian pdfs in calculating the mutual information of the beamformer outputs, and found the $K_{0}$ pdf to provide the best ASR performance on the separated speech.

We plan to develop an online version of the MMI beamforming algorithm presented here. This online algorithm will be capable of adjusting the active weight vectors $\mathbf{w}_{a, i}$ with each new snapshot in order to track changes of speaker position and movements of the speaker's head during an utterance.

\section{References}

[1] H. L. Van Trees, Optimum Array Processing, Wiley-Interscience, New York, 2002.

[2] Lucas C. Parra and Christopher V. Alvino, "Geometric source separation: Merging convolutive source separation with geometric beamforming," IEEE Trans. Speech Audio Proc., vol. 10, no. 6, pp. 352-362, September 2002.

[3] Craig Fancourt and Lucas Parra, "The generalized sidelobe decorrelator," in Proc. IEEE Workshop on Applications of Signal Processing to Audio and Acoustics, October 2001, pp. 167-170.

[4] M. Lincoln, I. McCowan, J. Vepa, and H.K. Maganti, "The multi-channel wall street journal audio visual corpus (mc-wsj-av): specification and initial experiments," in Proc. ASRU, November 2005, pp. 357-362.

[5] Aapo Hyvärinen and Erkki Oja, "Independent component analysis: Algorithms and applications," Neural Networks, vol. 13, pp. 411-430, 2000.

[6] J. McDonough and K. Kumatani, "Minimum mutual information beamforming," Tech. Rep. 107, Interactive Systems Lab, Universität Karlsruhe, August 2006.

[7] P. P. Vaidyanathan, Multirate Systems and Filter Banks, Prentice Hall, Englewood Cliffs, 1993.

[8] H. Buchner, R. Aichner, and W. Kellermann, "Blind source seperation for convolutive mixtures: A unified treatment," in Audio Signal Processing for Next-Generation Multimedia Communication Systems, pp. 255-289. Kluwer Academic, Boston, 2004.

[9] D. P. Bertsekas, Nonlinear Programming, Athena Scientific, Belmont, Massachusetts, 1995. 
[10] J. B. Allen and D. A. Berkley, "Image method for efficiently simulating small-room acoustics," Journal of the Acoustical Society of America, vol. 65, pp. 943-950, 1979.

[11] Helmut Brehm and Walter Stammler, "Description and generation of spherically invariant speechmodel signals," Signal Processing, vol. 12, pp. 119-141, 1987.

[12] Yudell L. Luke, The Special Functions and their Approximations, Academic Press, New York, 1969.

[13] Stephen Wolfram, The Mathematica Book, Cambridge University Press, Cambridge, 3 edition, 1996.

[14] T. Gehrig and J. McDonough, "Tracking and far-field speech recognition for multiple simultaneous speakers," Proc. Workshop on Machine Learning and Multimodal Interaction, September 2006.

[15] M.C. Wölfel and J.W. McDonough, "Minimum variance distortionless response spectral estimation, review and refinements," IEEE Signal Processing Magazine, vol. 22, no. 5, pp. 117-126, Sept. 2005.

[16] K. Fukunaga, Introduction to Statistical Pattern Recognition, Academic Press, New York, 1990.

[17] M. J. F. Gales, "Semi-tied covariance matrices," in Proc. ICASSP, 1998.

[18] G. Saon, D. Povey, and G. Zweig, "Anatomy of an extremely fast LVCSR decoder," in Proc. Interspeech, Lisbon, Portugal, 2005.

[19] A. Ljolje, F. Pereira, and M. Riley, "Efficient general lattice generation and rescoring," in Proc. Eurospeech, Budapest, Hungary, 1999.

[20] Jeroen Fransen, Dave Pye, Tony Robinson, Phil Woodland, and Steve Young, "Wsjcam0 corpus and recording description," Tech. Rep. CUED/F-INFENG/TR.192, Cambridge University Engineering Department (CUED) Speech Group, September 1994.

[21] J. Deller, J. Hansen, and J. Proakis, Discrete-Time Processing of Speech Signals, Macmillan Publishing, New York, 1993.

[22] T. Anastasakos, J. McDonough, R. Schwarz, and J. Makhoul, "A compact model for speakeradaptive training," in Proc. ICSLP, 1996, pp. 1137-1140.

[23] L. Uebel and P. Woodland, "Improvements in linear transform based speaker adaptation," in Proc. ICASSP, 2001.

[24] M. Wölfel, "Mel-Frequenzanpassung der Minimum Varianz Distortionless Response Einhüllenden," Proc. of ESSV, 2003.

[25] M. J. F. Gales, "Maximum likelihood linear transformations for HMM-based speech recognition," Computer Speech and Language, vol. 12, 1998.

[26] C. J. Leggetter and P. C. Woodland, "Maximum likelihood linear regression for speaker adaptation of continuous density hidden markov models," Computer Speech and Language, vol. 9, pp. 171-185, April 1995.

[27] Jan Mark de Haan, Nedelko Grbic, Ingvar Claesson, and Sven Erik Nordholm, "Filter bank design for subband adaptive microphone arrays," IEEE Trans. Speech and Audio Proc., vol. 11, no. 1, pp. 14-23, January 2003. 